\title{
Inhibitory Activity of Pyrroloisoxazolidine Derivatives against Chlamydia trachomatis
}

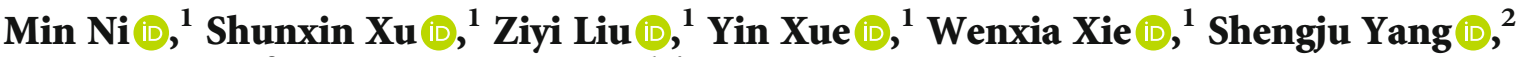 \\ Lingyan Liu $\mathbb{D}^{3}$ and Xiaofeng Bao $\mathbb{D D}^{1,4}$ \\ ${ }^{1}$ School of Pharmacy, Nantong University, Nantong 226001, China \\ ${ }^{2}$ Department of Dermatology and Venereology, Affiliated Hospital of Nantong University, Nantong 226001, China \\ ${ }^{3}$ State Key Laboratory of Elemento-Organic Chemsitry, Nankai University, Tianjin 300071, China \\ ${ }^{4}$ Key Laboratory of Inflammation and Molecular Drug Target of Jiangsu Province, Nantong University, Nantong 226001, China
}

Correspondence should be addressed to Shengju Yang; yangshengju80@163.com and Xiaofeng Bao; baoxi@ntu.edu.cn

Received 1 September 2020; Revised 26 January 2021; Accepted 1 March 2021; Published 15 March 2021

Academic Editor: Marcelo A. Soares

Copyright (C) 2021 Min Ni et al. This is an open access article distributed under the Creative Commons Attribution License, which permits unrestricted use, distribution, and reproduction in any medium, provided the original work is properly cited.

The obligate intracellular bacterium Chlamydia trachomatis is a group of worldwide human pathogens that can lead to serious reproductive problems. The frequent clinical treatment failure promoted the development of novel antichlamydial agents. Here, we firstly reported a group of pyrroloisoxazolidine-inhibited C. trachomatis in a dose-dependent manner in vitro. Among them, compounds 1 and 2 exhibited the strongest inhibitory activity with $\mathrm{IC}_{50}$ values from 7.25 to $9.73 \mu \mathrm{M}$. The compounds disturbed the whole intracellular life cycle of $C$. trachomatis, mainly targeting the middle reticulate body proliferation stages. Besides, the compounds partially inhibited the chlamydial infection by reducing elementary body infectivity at high concentration. Our findings suggest the potential of pyrroloisoxazolidine derivatives as promising lead molecules for the development of antichlamydial agents.

\section{Introduction}

Chlamydia trachomatis is the most prevalent bacterial pathogen that leads to sexually transmitted diseases (STD) in the world $[1,2]$. The serotypes $\mathrm{D}-\mathrm{K}$ are associated with urogenital tract infection, resulting in serious complications including ectopic pregnancy, abortion, infertility, and pelvic inflammatory disease in women $[2,3]$. The invasive serovars L1-L3 can cause lymphogranuloma venereum (LGV) that can lead to serious disseminated infections of the lymphatic system [2, 3]. In the United States, among the 2.3 million STD cases reported to the Centers for Disease Control and Prevention (CDC) in 2017, C. trachomatis infection remained the most common condition reported. More than 1.7 million cases were diagnosed as C. trachomatis infection, with 45 percent among 15 - to 24 -year-old females. In addition, the serovars A-C infect the ocular mucosa causing trachoma, the leading cause of preventable infectious blindness in many of the undeveloped countries $[4,5]$.
Chlamydia trachomatis is an obligate intracellular bacterium characterized by a highly specialized biphasic developmental cycle, morphologically switch between the extracellular infectious but nonproliferative elementary body (EB) and the intracellular replicative but noninfectious reticulate body $(\mathrm{RB})$. The infection is initiated by EB binding to and invading the host cell. Within the following 6-8h, internalized EB starts to transcript early genes and differentiates into RB inside the specialized vacuole inclusion. Subsequently, replicative $\mathrm{RB}$ divides by binary fission before redifferentiating back to infectious EB. Most chlamydial developmental cycles are complete in 40-72 hours when EBs are released from the host cell by either inclusion extrusion or cell lysis $[1,6,7]$.

Currently, uncomplicated C. trachomatis infections are commonly treated with either a single dose of azithromycin or doxycycline twice a day for at least 7 days $[8,9]$. Unfortunately, as the development of antibiotic-resistant bacteria, relapsing symptoms and treatment failures are frequently 
found after treatment with these first-choice antibiotics, resulting in Chlamydia persistence or long-term infection [10-12]. During the past few decades, plenty of efforts have been focused on vaccine development. However, no effective vaccine is available to prevent $C$. trachomatis infections up to date [13-15]. All these negative situations raise the pressing need to search for novel antichlamydial agents from natural materials or synthesized chemicals [16-25]. Isoxazolidine derivatives are a group of chemicals that possess a variety of biological and pharmaceutical activities including neuroactive, antioxidant, antibacterial, antifungal, and antiretroviral properties [26-30]. In this study, we firstly reported the antichlamydial activity of our previously synthesized eighteen pyrroloisoxazolidines via the hydroamination cyclization-[2+3]-cycloaddition with homopropargylic amines and nitrones using metal catalysts AgOAc [31]. We revealed the fact that these compounds affected the intracellular proliferation period of C. trachomatis, mainly on the middle RB proliferation stages.

\section{Materials and Methods}

2.1. Pyrroloisoxazolidine Derivatives. The pyrroloisoxazolidine derivatives 1-18 were synthesized and isolated as previous [31]. Compounds were dissolved in DMSO and were diluted to the appropriate concentrations using culture medium. To eliminate the interference of possible aggregates in diluted samples that might cause nonspecific inhibition against Chlamydia [32,33], culture medium containing individual compound was centrifuged for $30 \mathrm{~min}$ at $12700 \mathrm{rpm}$ $(18213 \mathrm{~g})$ at room temperature. The supernatant was then used to assess the antichlamydial activity as described in the following.

2.2. Chlamydia Strains and Culture Condition. Chlamydia trachomatis serovars D (strain UW-3/CX) and L2 (strain 434/Bu) were purchased from ATCC. HeLa cells purchased from ATCC were routinely cultured in DMEM supplemented with $10 \%$ heat-inactivated FBS (Sigma-Aldrich) and $10 \mu \mathrm{g} / \mathrm{mL}$ gentamicin at $37^{\circ} \mathrm{C}$ in a humidified atmosphere of $5 \%$ and were used for chlamydial propagation and cell culture experiments. EBs were purified by Renografin density gradient and stored in sucrose-phosphateglutamate (SPG) buffer at $-80^{\circ} \mathrm{C}$ [20]. A mouse polyclonal anti-MoPn (C. muridarum strain Nigg II) antibody, which was produced in our laboratory and cross-reacting with $C$. trachomatis D and L2, was used as a primary antibody for both stains detection [20].

2.3. Inclusion Body Immunofluorescence Staining. Cell monolayers seeded onto coverslips in a 24 -well plate were infected with C. trachomatis at a multiplicity of infection (MOI) of 0.2 inclusion-forming units (IFU) per cell. Unless noted, individual compound was added into culture medium at indicated concentrations simultaneously at the time of inoculation. $0.5 \%$ DMSO was used as negative control and $11.25 \mu \mathrm{M}$ tetracycline (Sigma-Aldrich) as positive control [20]. For C. trachomatis D, an additional centrifugation $(900 \times \mathrm{g}$ at room temperature for $1 \mathrm{~h}$ ) was applied to facilitate the infection.
At 36 hours postinfection (hpi), infected cells were fixed by precooled methanol and then subjected to sequential staining processed with a primary antibody and a FITC-conjugated secondary antibody. Evans blue was used as cytoplasm counterstain. Images were shot by an Olympus IX51 fluorescence microscope.

2.4. Infectious Progeny Inhibition Assay. Cells monolayers seeded in 48-well plate were infected and treated with chemicals as above. After incubation for 36 hours, infected cells were washed twice, scraped off, and lysed by sonication to release the infectious progeny EBs. Then the lysates were used to reinfect fresh cell monolayers grown in 96-well plate following 1:10 serial dilution. The inclusion counts were obtained by immunofluorescence staining as above to calculate the number of infectious progeny EBs in each sample. Percent inhibition was calculated based on EB counts in compound-treated samples relative to DMSO-treated negative control.

2.5. WST-1 Cell Viability. Uninfected HeLa cells were cultured with $32 \mu \mathrm{M}$ (the highest concentration used in antichlamydial analysis) of individual compound or $0.5 \%$ DMSO for $48 \mathrm{~h}$. After $10 \mu \mathrm{L}$ of WST-1 reagent was added, cells were incubated for an additional $1 \mathrm{~h}$, and absorbance at $450 \mathrm{~nm}$ was recorded. The viability of compound-treated cells was calculated by the absorbance relative to that of DMSO control setting as $100 \%$ [22].

2.6. Host Cell Cytotoxicity. Uninfected HeLa cells were cultured with indicated concentration of individual compound or $0.5 \%$ DMSO. Forty-eight hours later, cells were fixed with methanol and stained with Evans blue for morphology or DAPI for nuclear integrity. Otherwise, at $48 \mathrm{~h}$ later, cells were detached and enumerated by a hemacytometer [20].

2.7. Host Cell Pretreatment Assay. Cells seeded in 48-well plate were incubated with 2,8 , or $32 \mu \mathrm{M}$ individual compound at $37^{\circ} \mathrm{C}$ for indicated times. After twice washing with SPG to remove residual compound, cells were infected with EBs at an MOI of 0.2. At $36 \mathrm{hpi}$, the inclusion count in each well was determined by immunofluorescence staining as above.

2.8. Elementary Body Pretreatment Assay. Chlamydia trachomatis L2 EBs suspended in SPG at $4 \times 10^{5} \mathrm{IFU} / \mathrm{mL}$ were exposed to 2,8 , or $32 \mu \mathrm{M}$ individual compound at $37^{\circ} \mathrm{C}$ or $4^{\circ} \mathrm{C}$ for indicated times. EBs then were washed twice to eliminate the residual chemical and were immediately loaded onto HeLa cell monolayers at an MOI of 0.2. The infective EB count was measured by quantifying inclusion numbers at 36 hpi using immunofluorescence staining as above.

2.9. Later Treatment Assay. HeLa cells seeded in 48-well plate were infected with C. trachomatis L2 at an MOI of 0.2 and were treated with 2,8 , or $32 \mu \mathrm{M}$ of compound at $0,2,12$, and $24 \mathrm{hpi}$. At $36 \mathrm{hpi}$, cells were collected, and the production of infectious progeny EBs was determined as described above. Percent inhibition was calculated based on progeny 
EB count in compound-treated samples relative to DMSOtreated negative control.

2.10. Withdrawal Assay. HeLa cells seeded in 48-well plate were infected with C. trachomatis L2 at an MOI of 0.2 and simultaneously treated with 2,8 , or $32 \mu \mathrm{M}$ of compound. At 2, 12, 24, and $36 \mathrm{hpi}$, infected cells were washed twice to remove residual inhibitor, and fresh medium was added. At $36 \mathrm{hpi}$, the production of infectious progeny EBs and percent inhibition data were determined as above.

2.11. Period Incubation Assay. HeLa cells seeded in 48-well plate were infected with $C$. trachomatis L2 at an MOI of 0.2 and treated with 2,8 , or $32 \mu \mathrm{M}$ of compound during $0-2,2$ $12,12-24$, and $24-36 \mathrm{~h}$ period. At $36 \mathrm{hpi}$, the production of infectious progeny EBs and percent inhibition data were determined as above.

2.12. Statistical Analysis. Data from the three independent experiments were presented as mean \pm standard deviations (SD) and were subjected to pairwise Student's $t$-tests. $p<$ 0.05 and $p<0.01$ are considered statistically significant and statistically highly significant, respectively. The $\mathrm{IC}_{50}$ values, defined as the concentration of compound at which the generation of infectious progeny EBs was reduced by $50 \%$ relative to negative control, were calculated by nonlinear regression from percent inhibition data using the GraphPad Prism 5 software, and presented as mean (95\% confidence interval).

\section{Results}

3.1. Pyrroloisoxazolidines Inhibited C. trachomatis in a DoseDependent Manner. The antichlamydial activity of eighteen synthetic pyrroloisoxazolidines was screened by determining their inhibitory effect on the generation of infectious progeny EBs at concentration doubly increased from 1 to $32 \mu \mathrm{M}$ against $C$. trachomatis $\mathrm{D}$ and $\mathrm{L} 2[22,34]$. The $\mathrm{IC}_{50}$ values were calculated from percent inhibition data and presented in Table 1. All the compounds inhibited both C. trachomatis with $\mathrm{IC}_{50}$ values lower than $32 \mu \mathrm{M}$ for at least one tested strain. Among them, compounds 1 and 2 showed the best inhibitory activity with similar $\mathrm{IC}_{50}$ values slightly lower than $10 \mu \mathrm{M}$ for both strains. For C. trachomatis D, compounds 4 , 5 , and 7 exhibited $\mathrm{IC}_{50}$ values higher than $32 \mu \mathrm{M}$, and those were compounds $9,11,13,17$, and 18 for C. trachomatis L2. All other compounds possessed $\mathrm{IC}_{50}$ values between 10 and $32 \mu \mathrm{M}$.

As obligate intracellular pathogens, C. trachomatis relied on the host cells for survival and replication. To exclude the possibility of cytotoxicity that disturbing chlamydial growth and displayed as compounds' antichlamydial activity, the cytotoxic effect of compounds on host cells was assessed using the WST-1 method by treatment of uninfected HeLa cells with $32 \mu \mathrm{M}$ of individual compound, the highest concentration used in our antichlamydial analysis. As presented in Table 1, most compounds did not affect the proliferation activity of HeLa cells, except three chemicals $(6,7$, and 10) that weakly decreased the cell viability to about $90 \%$ of DMSO control. Because compounds 1 and 2 have the highest inhibitory activity but not cytotoxicity, they were chosen for further antichlamydial mechanism analysis.

To understand how the compounds inhibited C. trachomatis, the direct inhibitory effect of compounds 1 and 2 was evaluate using immunofluorescence staining by culturing C. trachomatis L2-infected cells containing individual compound. Compared with the negative control $(0.5 \%$ DMSO), both compounds inhibited the formation (number) and growth (size) of C. trachomatis L2 inclusions in a dosedependent manner (Figure 1(a)). At the highest concentration of $32 \mu \mathrm{M}$, inclusions were few and tiny, analogous to the positive control tetracycline (final concentration, $11.25 \mu \mathrm{M}$ ) [19]. In addition, the reduction on the production of progeny EBs was consistent with that of the inclusion number and size (Figure 1(b)). The direct inhibitory effect was also analyzed for C. trachomatis D. Happened as expected, changes in inclusion size and number and the progeny production that response to both chemicals are highly similar to those observed for C. trachomatis L2, with slightly weaker effect (Figures 1(a) and 1(c)).

As shown in Figure 1(a), the morphology of infected HeLa cells was well preserved when treated with compound. To further verify that compounds 1 and 2 are not toxic to host cells, the uninfected HeLa cells were treated with individual compound for $48 \mathrm{~h}$, and then their morphology, nuclear integrity, and cell proliferation were measured [20]. No substantial cytotoxic activity on host cells was observed from morphological features by Evans blue staining, nuclear integrity by DAPI staining, and cell proliferation by counting cell numbers (Figure 2). Coupled with the WST-1 data (Table 1), these findings verified that compounds 1 and 2 do not affect host cells, boosting the conclusion that the antichlamydial activity of compounds is not through host cell toxicity.

3.2. Pyrroloisoxazolidines Weakly Attenuated the EB Infectivity. Chlamydia trachomatis has a unique $\mathrm{EB} / \mathrm{RB}$ biphasic life cycle, which offers the infection steps and the intracellular proliferation period for therapeutic intervention. To explore whether the compounds affected the chlamydial infection steps, the HeLa cells or EBs were pretreated with compound prior to infection, and then the influence on EB entry efficiency was analyzed by counting inclusion numbers. Due to the relative easier for culture and shorter developmental cycle, C. trachomatis L2 is commonly represented as a laboratory model for C. trachomatis in cell culture research and is picked for our subsequent mechanism study.

As shown in Figure 3, pretreatment of HeLa cells with 2, 8 , and $32 \mu \mathrm{M}$ of compound 1 or 2 prior to infection for $1 \mathrm{~h}$ did not reduce the formation of chlamydial inclusions. The inclusion number did not change even extending the incubation time to 4 hours, indicating that both compounds did not affect the host cell during infection process. Because EBs suffered a loss of infectivity at $37^{\circ} \mathrm{C}$ in SPG buffer containing $0.5 \%$ DMSO (Figure 4 ), $4^{\circ} \mathrm{C}$ EB pretreatment was supplemented to the physiological temperature. When EBs were pretreated with 2,8 , and $32 \mu \mathrm{M}$ of compound for $1 \mathrm{~h}$, the EB counts that enter into host cells did not change at both 
TABLE 1: The $\mathrm{IC}_{50}$ values $(\mu \mathrm{M})$ and cell viability of pyrroloisoxazolidine-treated uninfected cells. $\mathrm{IC}_{50}$ values are presented as the mean $(95 \%$ confidence interval). * indicates statistically significant $(p<0.05)$ difference of cell proliferation activity between compound-treated and DMSO-treated cells.

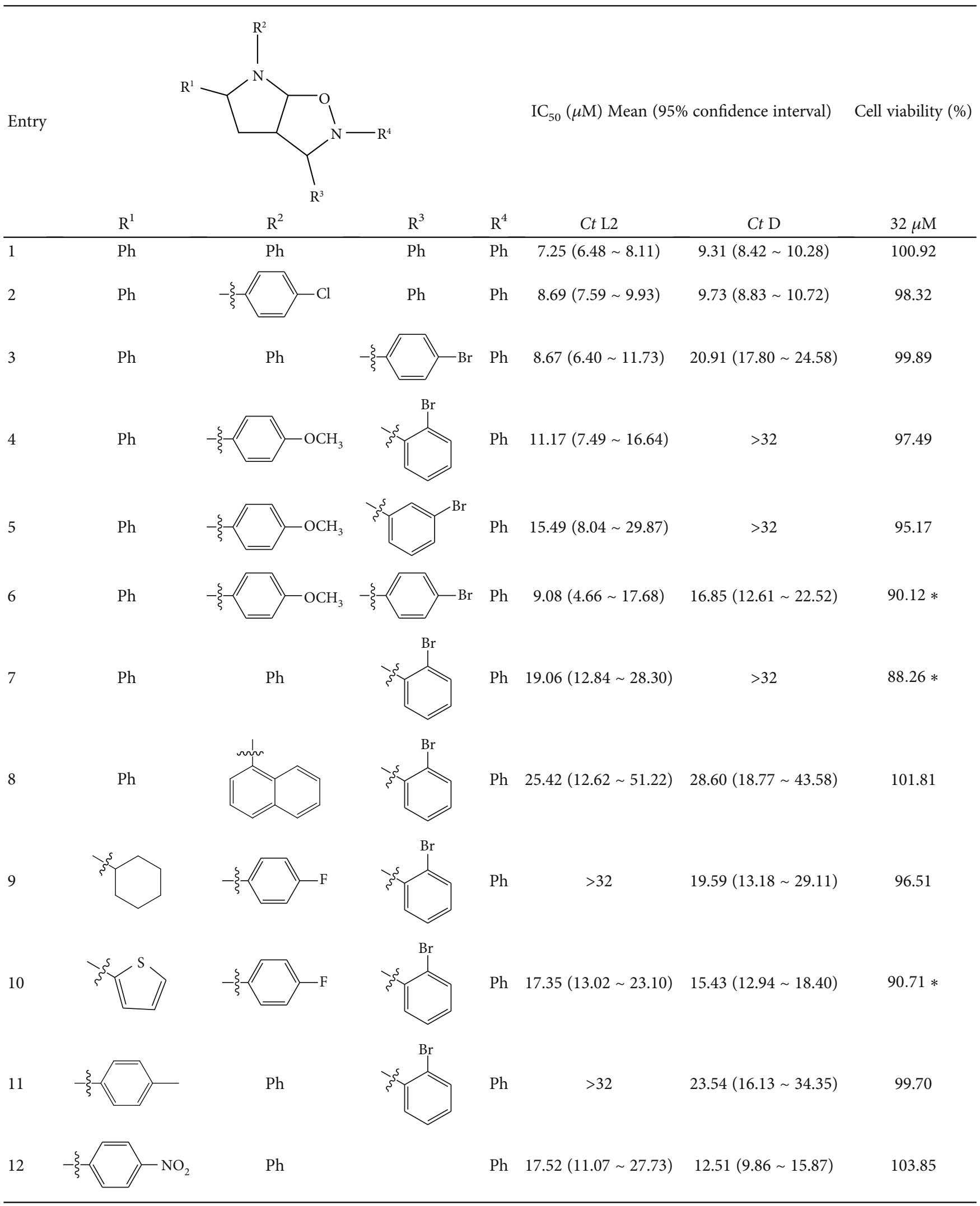


TABLE 1: Continued.

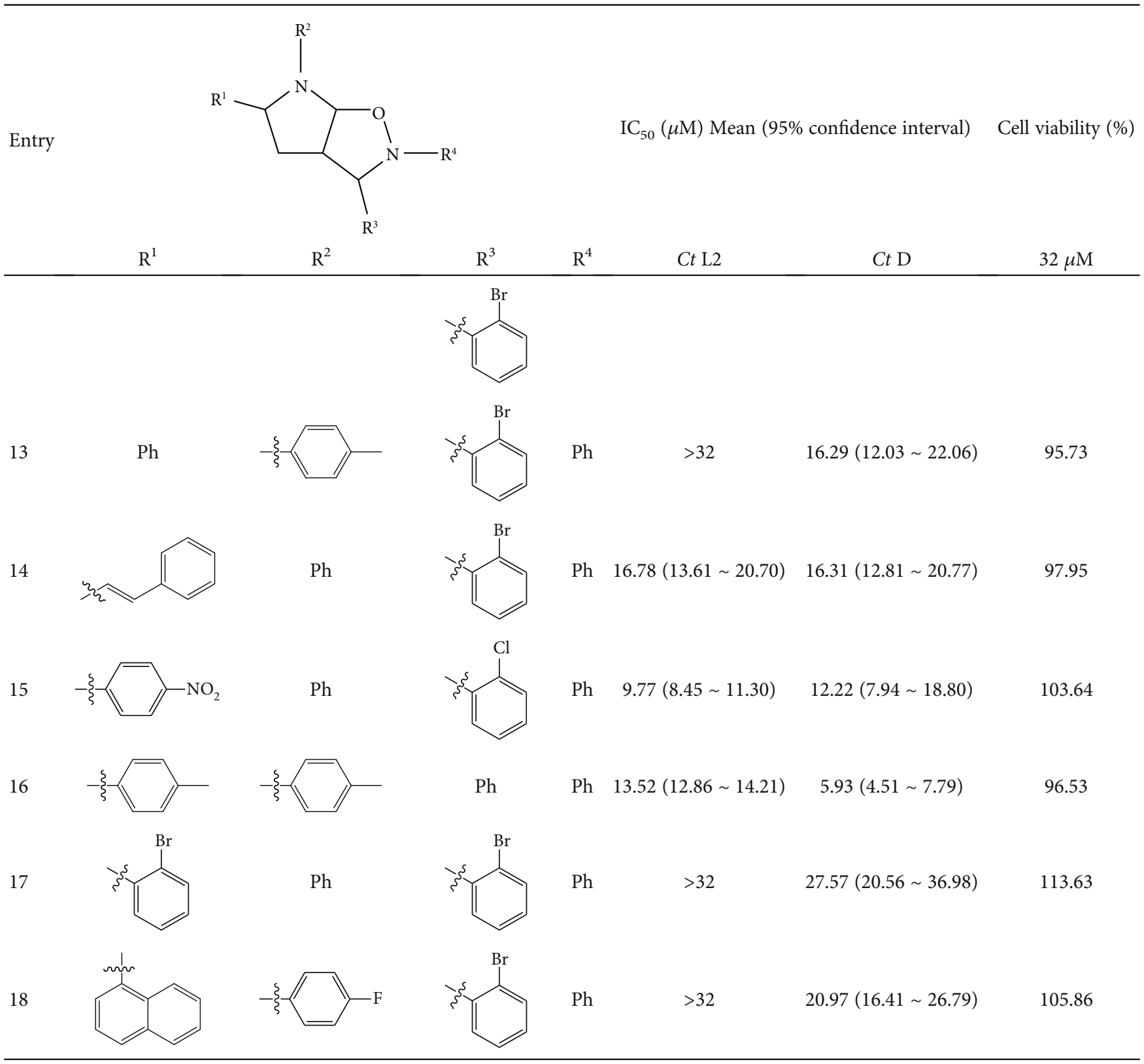

temperatures (Figure 4). However, if the EBs were pretreated with chemical for $4 \mathrm{~h}$, the EB entry efficiency slightly dropped at the highest $32 \mu \mathrm{M}$ for both compounds at $4^{\circ} \mathrm{C}$, revealing a mild interaction between the compound and EB that weakly attenuated the EB infectivity.

3.3. Pyrroloisoxazolidines Disturbed the Chlamydial Intracellular Life Cycle. Since the chlamydial infection process was not the primary target of the compounds, the intracellular proliferation cycle was analyzed. Firstly, the later treatment assay was used that the compound was added to infected cells at different times postinoculation, and then the production of infectious progenies was quantified at $36 \mathrm{hpi}$ (Figure 5(a)). As shown in Figures 5(b)-5(d), compared with 0 hpi sample (positive control, the cells were treated with compound for whole incubation period), the inhibitory activity for both compounds was maintained when chemical was added before 12 hpi. However, the inhibitory effect was dramatically dropped if the compound was added at $24 \mathrm{hpi}$, when the suppression activity was weak but still maintaining a substantial effect.

Secondly, the withdrawal assay was performed that the compound was added simultaneously with inoculation and then was removed at different times postinfection. The production of infectious progenies was quantified at 36 hpi to evaluate the effect of different treatment scheme (Figure 6(a)). As lower concentrations of 2 and $8 \mu \mathrm{M}$ (Figures 6(b) and 6(c)), compared with 36 hpi sample (positive control, the cells were treated with compound for whole infection period), the inhibitory capacity for both compounds was maintained when chemical was removed 


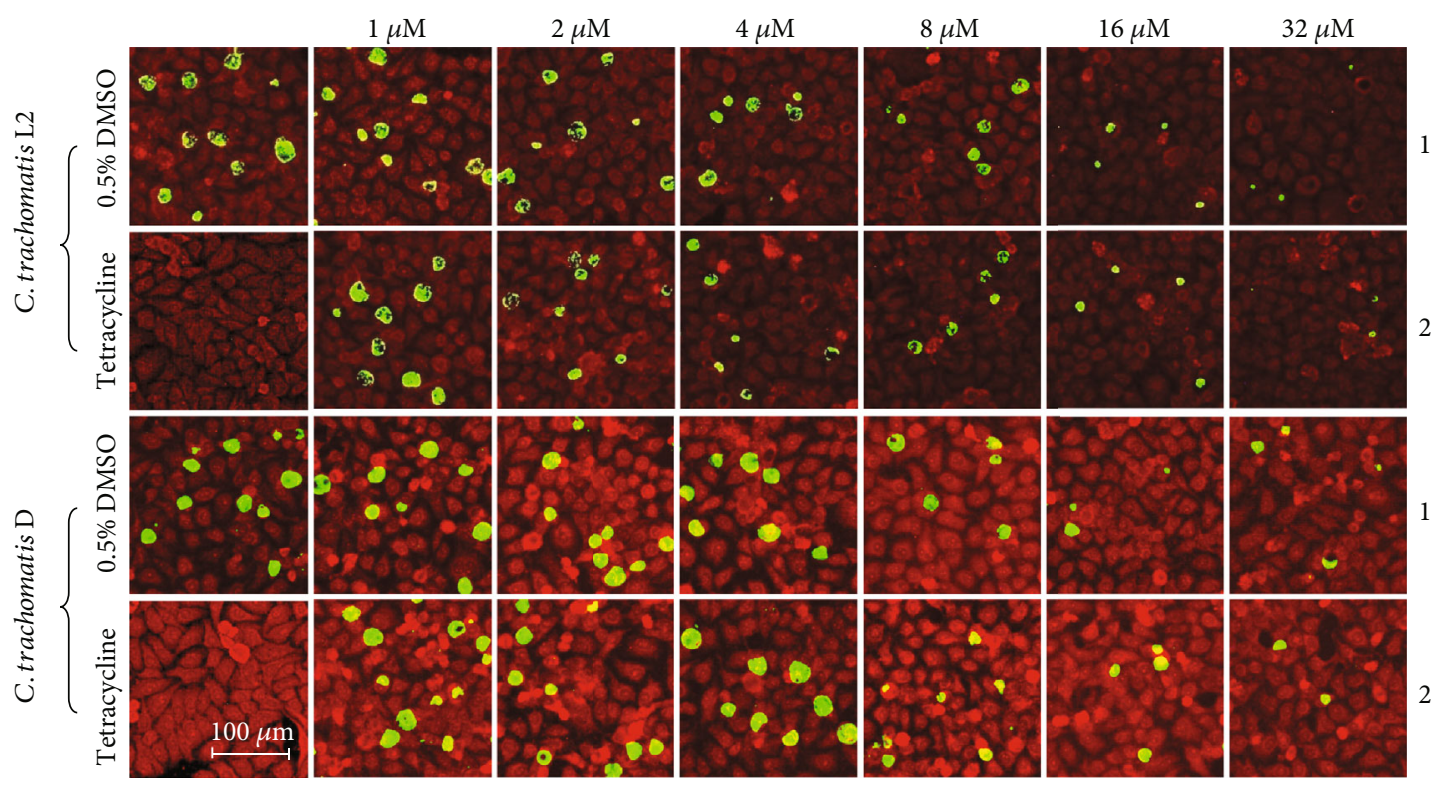

(a)

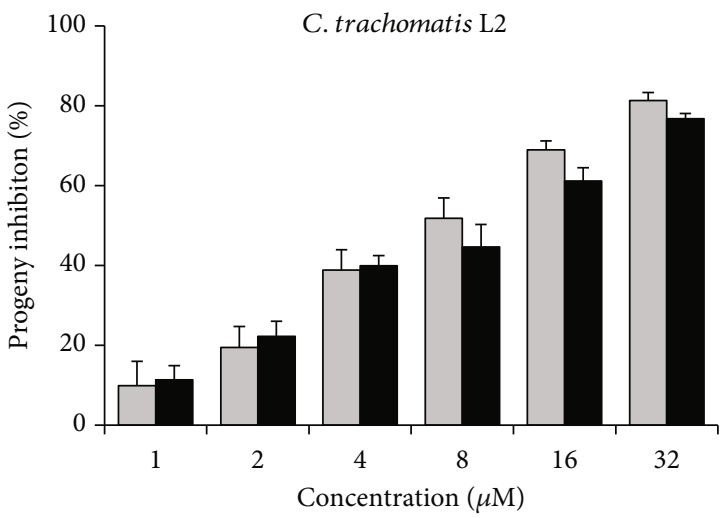

$\square 1$

a 2

(b)

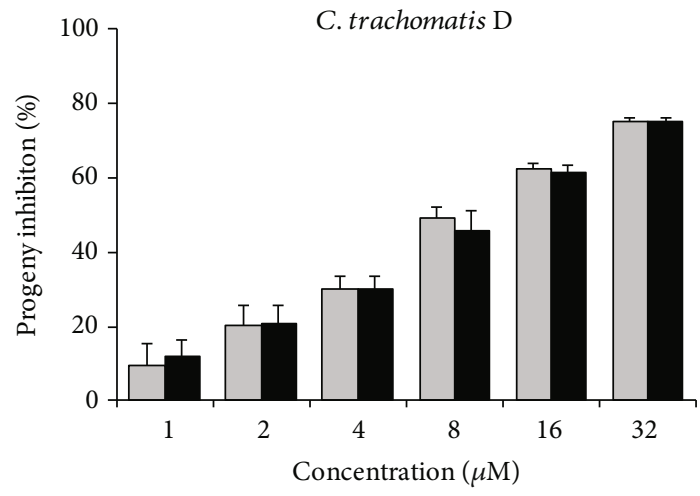

(c)

FIgure 1: Inhibition effect of compounds 1 and 2 on C. trachomatis. HeLa cells were infected with C. trachomatis L2 or D at an MOI of 0.2 in the presence of $0.5 \%$ DMSO, $11.25 \mu \mathrm{M}$ tetracycline, or various concentrations of individual compound. Cells were either fixed or lysed for determination of infectious progeny EBs at $36 \mathrm{hpi}$. (a) Representative immunofluorescence microscopy images (scale bar $=100 \mu \mathrm{m}$ ). Inclusions were stained with a polyclonal anti-MoPn antibody that cross-reacts with C. trachomatis L2 and D (green), and cells were counterstained with Evans blue (red). (b, c) The percent inhibition of production of infectious progeny EBs was calculated based on data in compound-treated samples relative to DMSO-treated control. Data are presented as mean \pm SD of three independent assays.

after 24 hpi. At highest $32 \mu \mathrm{M}$ (Figure 6(d)), the inhibition effect slightly dropped from approximately $80 \%$ to $75 \%$ at 24 hpi. For all concentrations tested, the inhibitory efficiency for both compounds was significantly reduced if the chemical was removed before 12 hpi. The earlier the compound was removed, the weaker inhibitory effect was obtained.

Finally, the period incubation assay was applied that the infected cells were treated with compound for indicated time periods, and then the production of infectious progenies was quantified at 36 hpi (Figure 7(a)). For all concentrations (Figures $7(\mathrm{~b})-7(\mathrm{~d})$ ), both compounds almost lost their antichlamydial activity with $0-2 \mathrm{~h}$ treatment except the highest $32 \mu \mathrm{M}$. They possessed the strongest inhibitory effect at $12-$
$24 \mathrm{~h}$ period close to the full-time positive control. Besides, both $2-12 \mathrm{~h}$ and $24-36 \mathrm{~h}$ treatment revealed similarly partial inhibition, slightly higher for $2-12 \mathrm{~h}$ period.

\section{Discussion}

Chlamydia trachomatis is a worldwide pathogen responsible for a variety of human diseases. Although it is susceptible to several broad-spectrum antibiotics, clinical treatment failure has happened frequently. Until now, there is no effective vaccine for human use. These facts necessitate the continuous efforts to discover and develop novel nonantibiotic antichlamydial agents [16-25]. 


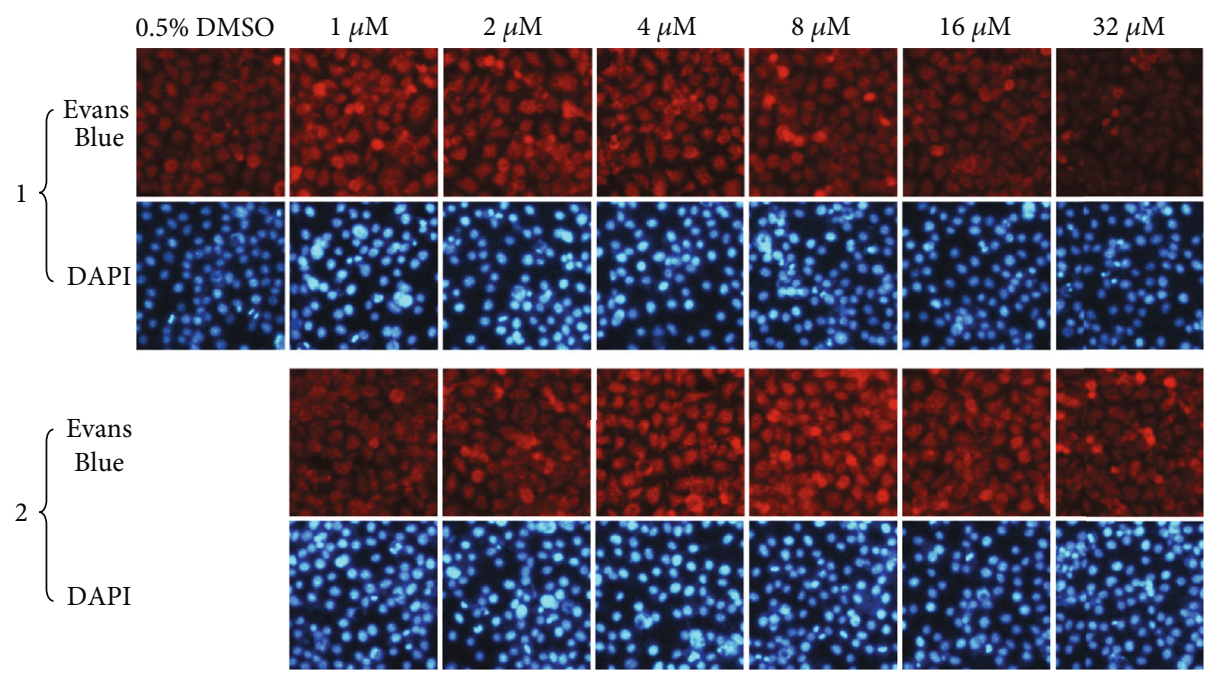

(a)

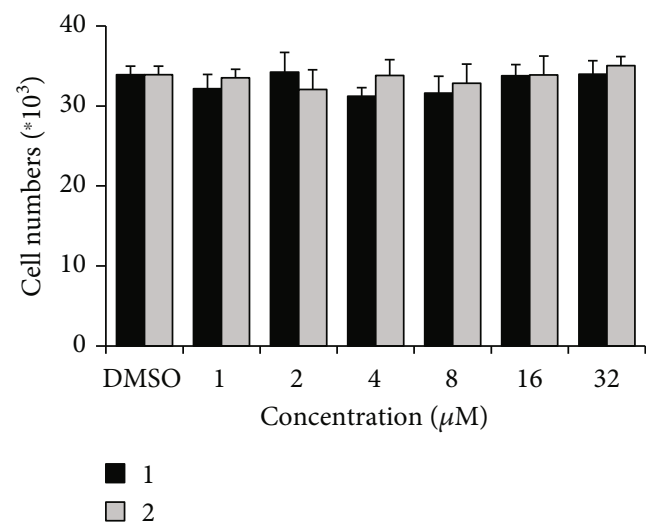

(b)

FIGURE 2: Lack of cytotoxicity of compounds 1 and 2 on HeLa cells. Uninfected HeLa cells were cultured with medium containing various concentrations of individual compound or $0.5 \%$ DMSO for $48 \mathrm{~h}$. (a) The cellular morphology was stained with Evans blue, and cell nucleus was stained with DAPI. (b) The cells were enumerated by a hemacytometer. Data are presented as mean \pm SD of three independent assays.

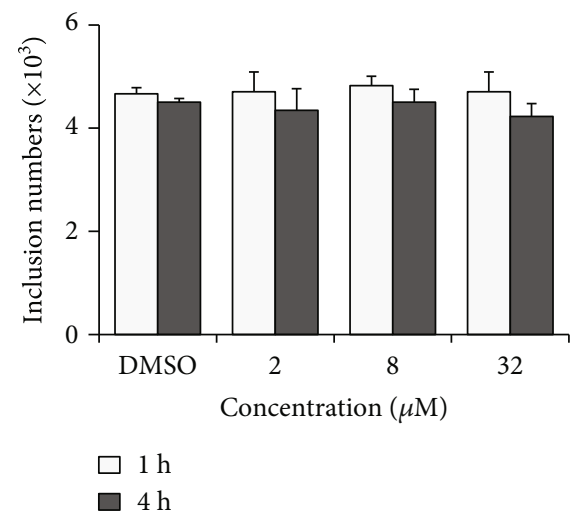

(a)

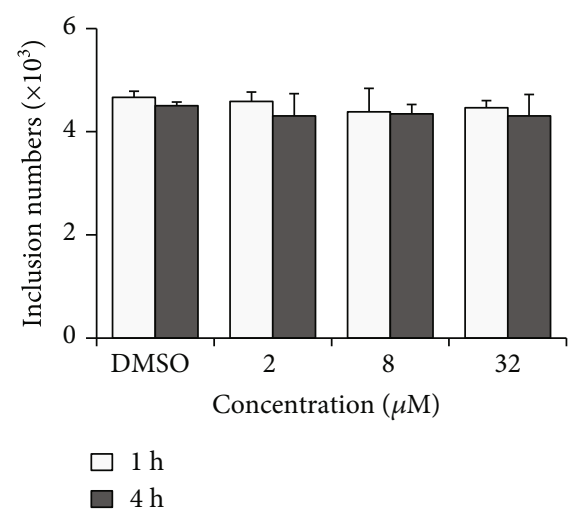

(b)

FIgURE 3: Host cells were not involved in the compounds' antichlamydial activity. HeLa cells were incubated with 2 , 8 , or $32 \mu \mathrm{M}$ compound 1 (a) or 2 (b) at $37^{\circ} \mathrm{C}$ for 1 or $4 \mathrm{~h}$. After washes, cells were infected with EBs. At 36 hpi, the inclusion was counted as described in "Materials and Methods." Data are presented as mean \pm SD of three independent assays. 

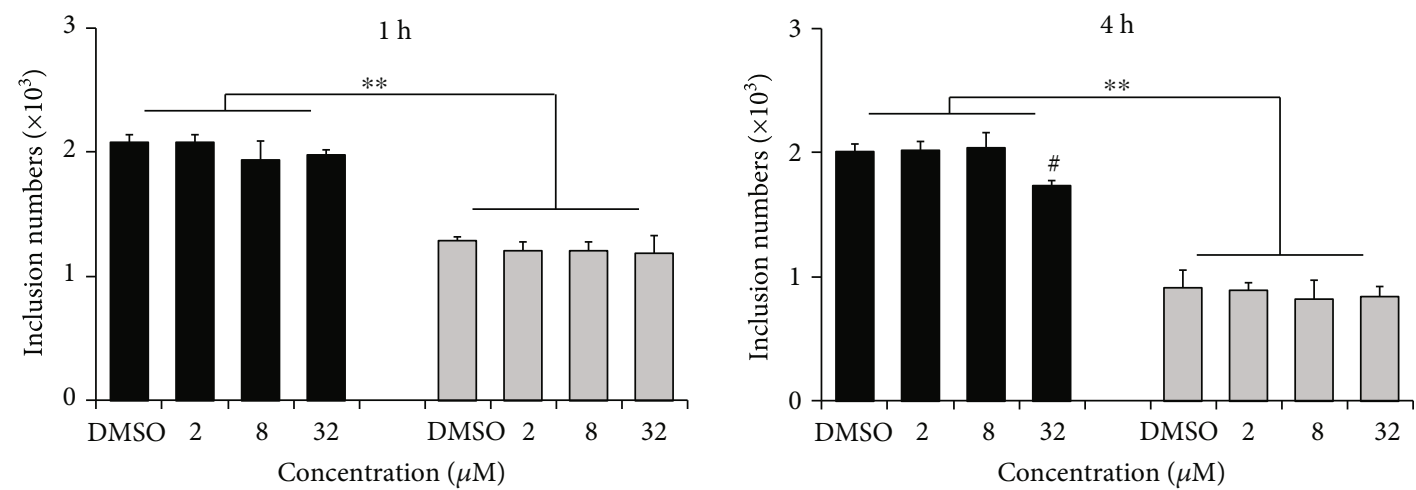

$$
\begin{aligned}
& \square 1 \mathrm{~h} \\
& \square 4 \mathrm{~h}
\end{aligned}
$$

(a)
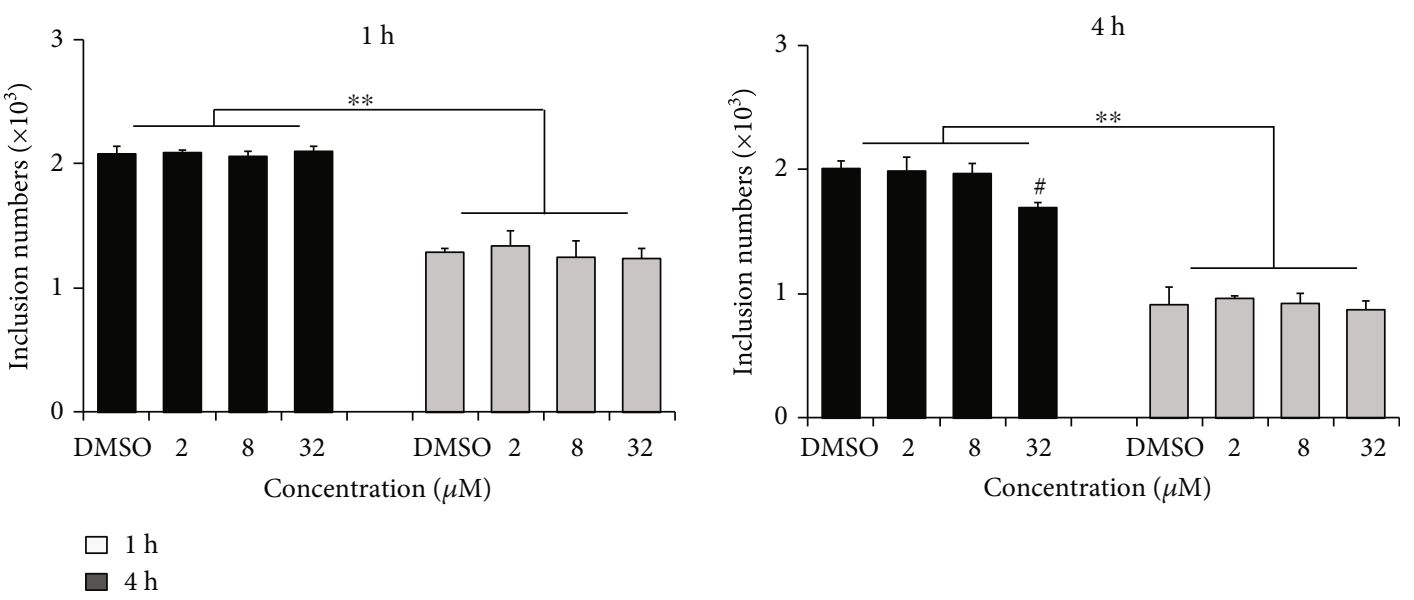

(b)

FIgURE 4: Compounds 1 and 2 weakly attenuated the EB infectivity. Chlamydia trachomatis L2 EBs were incubated with 2 , 8, or $32 \mu \mathrm{M}$ compound 1 (a) or 2 (b) at $37^{\circ} \mathrm{C}$ or $4^{\circ} \mathrm{C}$ for 1 or $4 \mathrm{~h}$. After washes, EBs were used to infect HeLa cell. At 36 hpi, the inclusion was counted as described in "Materials and Methods." Data are presented as mean \pm SD of three independent assays. $* * p<0.01$, compared $4^{\circ} \mathrm{C}$ with $37^{\circ} \mathrm{C}$ by Student's $t$-tests. ${ }^{*} p<0.05$, compared with each DMSO control sample by Student's $t$-tests.

In search for antichlamydial agents from the synthesized compounds, we firstly reported that a group of pyrroloisoxazolidines inhibited $C$. trachomatis propagation. The $\mathrm{IC}_{50}$ values from the yield of infectious progeny EBs against $C$. trachomatis $\mathrm{L} 2$ and $\mathrm{D}$ exhibited that most of the derivatives inhibited chlamydial growth in micromolar range (Table 1). Compounds 1 and 2 showed the best inhibitory activity with $\mathrm{IC}_{50}$ values in the range of 7.25 to $9.73 \mu \mathrm{M}$. The C-4 methoxyl group of phenyl on R2 position of compounds 4 and 5 was contributed to the weak antichlamydial activity on C. trachomatis $\mathrm{D}$. Compound 16 displayed the best inhibitory activity on C. trachomatis $\mathrm{D}$ with $\mathrm{IC}_{50}$ value of $5.93 \mu \mathrm{M}$, which indicated the C-4 methyl group of phenyl on both R1 and R2 favored its inhibitory activity. Compound 1 displayed the best inhibitory activity on C. trachomatis $\mathrm{L} 2$ with $\mathrm{IC}_{50}$ value of $7.25 \mu \mathrm{M}$, which indicated the substitute groups on phenyl was harmful to their inhibitory activity. These derivatives also showed no selectivity in their inhibitory activity against $C$. trachomatis $\mathrm{L} 2$ and $\mathrm{D}$, which suggested that they might inhibit both Chlamydia strains through the same mechanism.
WST-1 data showed that at $32 \mu \mathrm{M}$, the highest concentration used in our antichlamydial assays, most compounds had no apparent effects on the host cell viability except compounds 6,7 , and 10 which weakly decreased the cell viability to about $90 \%$ of DMSO control (Table 1). The compounds 1 and 2, the derivatives with the best antichlamydial activity, did not show cytotoxicity using the WST-1 method. In addition, the cellular morphology, nuclear integrity, and cell proliferation data also eliminated the potential toxicity of compounds 1 and 2 that may obstruct chlamydial growth (Figure 2), easily coming to a conclusion that the antichlamydial activity of the compounds is not related to their cytotoxicity.

The developmental cycle of $C$. trachomatis begins with infection process as adhesion and entry of the EB into a host cell, relying on multiple redundant factors both on the host cell and bacteria surface [35]. Pretreatment of HeLa cells with compound 1 or 2 could not impair the EB counts that enter into cells (Figure 3), excluding the roles of host cell factors that involved in the compounds' antichlamydial activity. Lower concentration and shorter time incubation of EBs with 


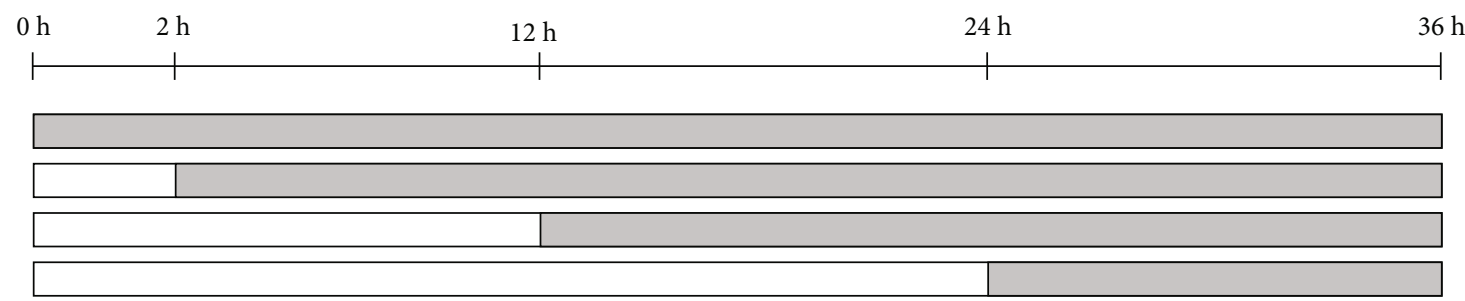

(a)

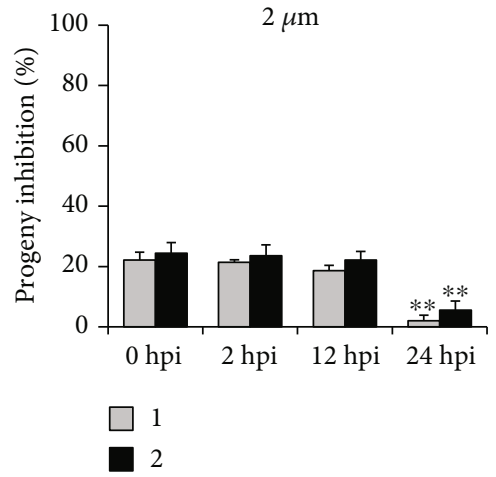

(b)

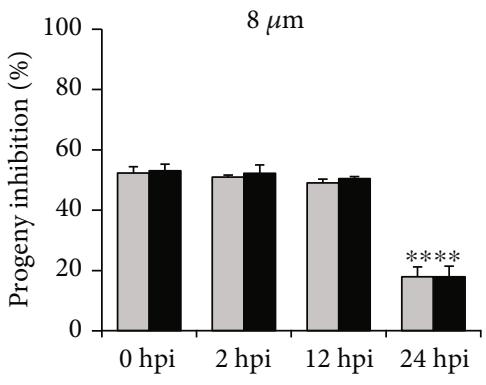

(c)

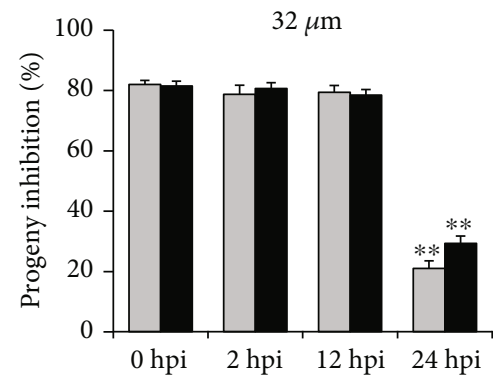

(d)

FIGURE 5: Later treatment assay. (a) HeLa cells infected with C. trachomatis L2 were treated with 2, 8, or $32 \mu \mathrm{M}$ individual compound at 0 , 2 , 12, or $24 \mathrm{hpi}$. The production of progeny EBs was determined at $36 \mathrm{hpi}$. (b-d) Percent inhibition of progeny EBs was calculated based on data in compound-treated samples relative to DMSO-treated control. Data are presented as mean \pm SD of three independent assays. $* * p<0.01$, compared with each 0 hpi positive control sample by Student's $t$-tests.

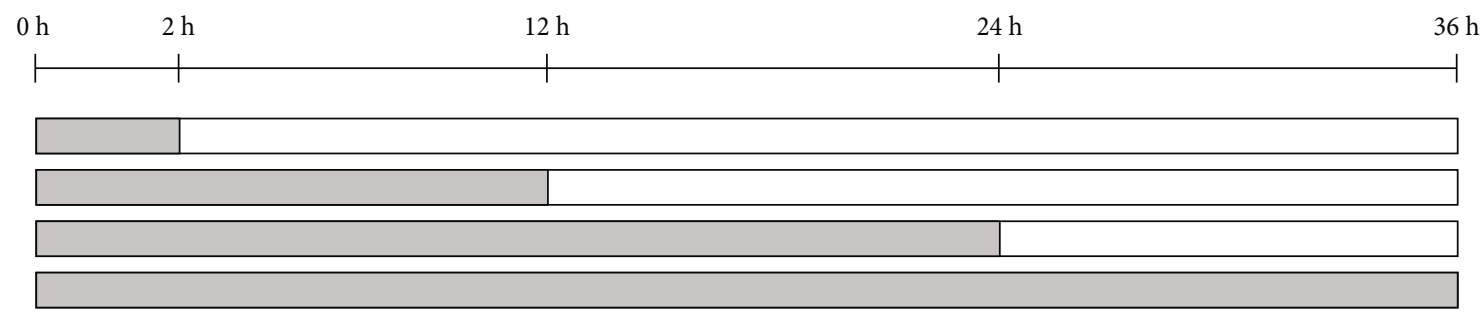

(a)
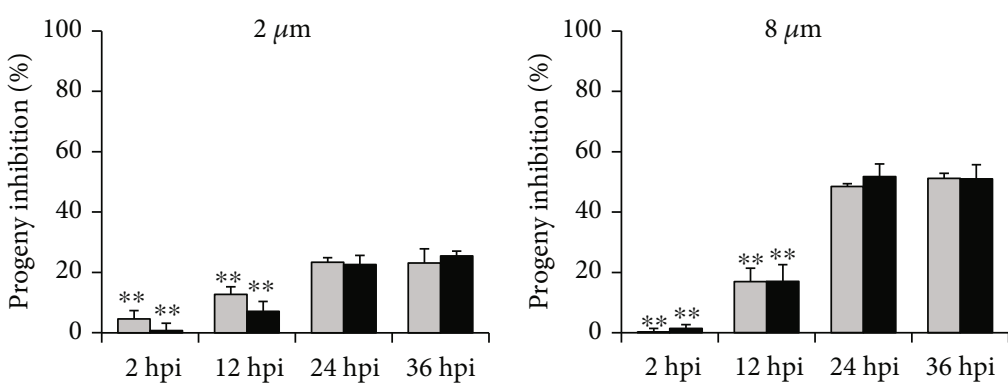

(c)

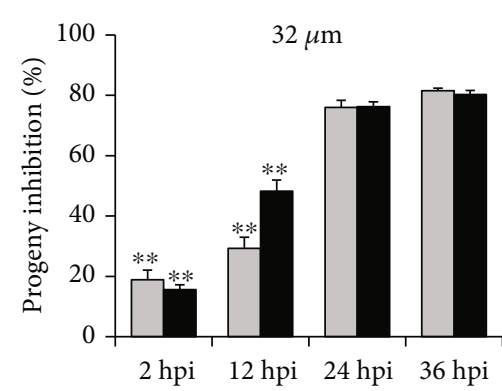

(d)

FIgURE 6: Withdrawal assay. (a) HeLa cells infected with C. trachomatis L2 were simultaneously treated with 2 , 8 , or $32 \mu \mathrm{M}$ individual compound. The compound was removed at 2, 12, 24, or $36 \mathrm{hpi}$, and the production of progeny EBs was determined at 36 hpi. (b-d) Percent inhibition of progeny EBs was calculated based on data in compound-treated samples relative to DMSO-treated control. Data are presented as mean $\pm \mathrm{SD}$ of three independent assays. $* p<0.05, * * p<0.01$, compared with each 36 hpi positive control sample by Student's $t$-tests. 


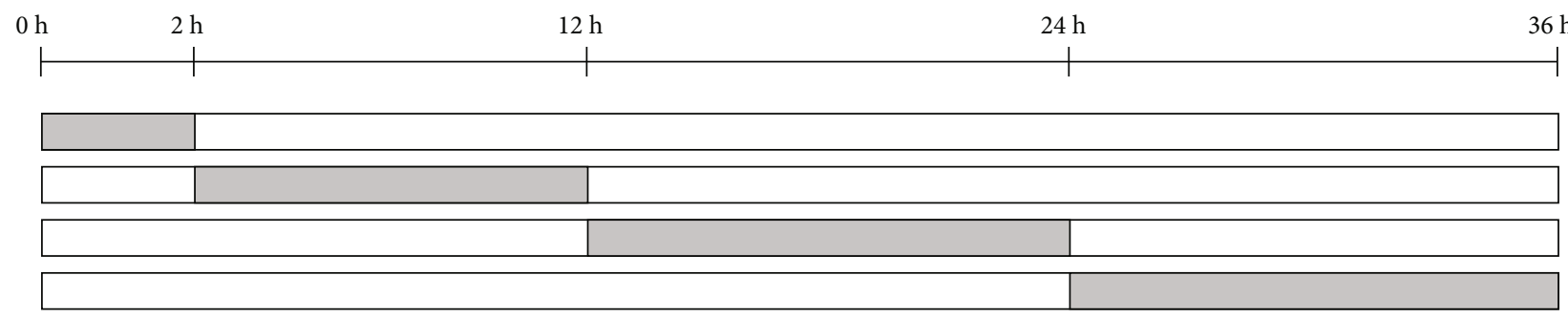

(a)

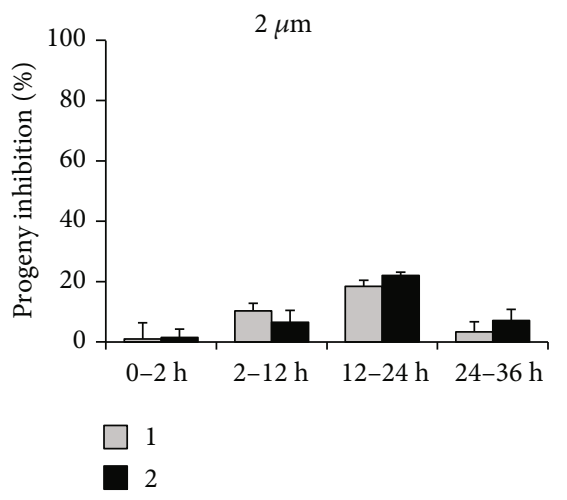

(b)

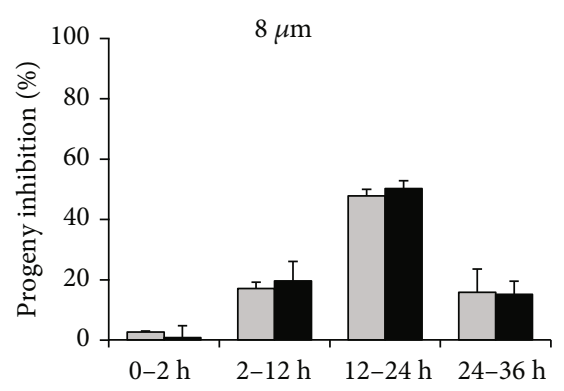

(c)

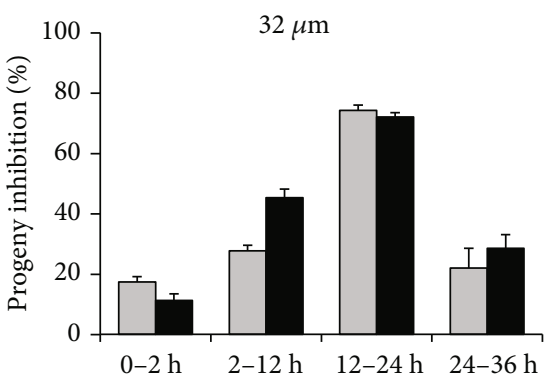

(d)

Figure 7: Period incubation assay. (a) HeLa cells infected with C. trachomatis L2 were treated with 2, 8, or $32 \mu \mathrm{M}$ individual compound for indicated times. The production of progeny EBs was determined at $36 \mathrm{hpi}$. (b-d) Percent inhibition of progeny EBs was calculated based on data in compound-treated samples relative to DMSO-treated control. Data are presented as mean \pm SD of three independent assays.

compound 1 or 2 also did not affect the entered EB counts (Figure 4). However, when the chemical concentration was raised to $32 \mu \mathrm{M}$ and the incubation time was extended to 4 hours, the EB infectivity was weakly but statistically decreased (Figure 4). This result was verified by the period incubation assay that $32 \mu \mathrm{M}$ compound 1 or 2 possessed a weak inhibitory effect if treated at $0-2 \mathrm{~h}$ (Figure $7(\mathrm{~d})$ ), the EB binding and invading stages. These combined data suggested the potential effect of compounds on bacteria surface factors that are related to the attachment/entry of C. trachomatis into host cells to reduce EB infectivity, which might be partially concerned in the inhibitory effect of the compounds.

The compound pretreatment only weakly impaired EBs infectivity, while the cotreatment condition almost fully blocked chlamydial growth (Figure 1), pointing out the speculation that the compounds affected the intracellular stages of the chlamydial life cycle. According to the later treatment assay, the antichlamydial activity of compounds 1 and 2 was maintained when chemical was added before 12 hpi while it was dramatically dropped if added at 24 hpi (Figure 5), indicating the strong inhibition on the intracellular period of $C$. trachomatis after 12 hpi. Meanwhile, the withdrawal assay data revealed that the inhibitory effect of compounds 1 and 2 was relatively well preserved when chemical was removed after 24 hpi while it was significantly reduced if removed before $12 \mathrm{hpi}$ (Figure 6), suggesting the strong intracellular proliferation inhibition before 24 hpi. Based on these two results, a conclusion was easily deduced that the compounds primarily affected the middle $12-24 \mathrm{~h}$ period of C. trachomatis life cycle, when the $\mathrm{RB}$ was replicated during this phase. This conclusion was well documented by the observation that the production of infectious progenies was significantly inhibited when the compound 1 or 2 was added during the $12-24 \mathrm{~h}$ period (Figure 7 ), which was close to the 0 $36 \mathrm{~h}$ full-time positive control (Figures 5 and 6).

Compounds 1 and 2 kept a weak but substantial inhibitory effect when added at 24 hpi (Figure 5). These results suggested that the intracellular life cycle of $C$. trachomatis after 24 hours could be disturbed by the compounds although it was not that powerful, confirmed by the $24-36 \mathrm{~h}$ period incubation when the infectious progeny EBs were partially inhibited (Figure 7 ). Furthermore, the $2-12 \mathrm{~h}$ period of intracellular chlamydial growth was also partially prevented by compounds 1 and 2 (Figure 7), verified by the 12 hpi withdrawal data (Figure 6).

\section{Conclusions}

In summary, we firstly identified the antichlamydial activity of a group of pyrroloisoxazolidines in vitro. Among them, compounds 1 and 2 exhibit the greatest inhibitory activity with $\mathrm{IC}_{50}$ values in the range of 7.25 to $9.73 \mu \mathrm{M}$. All combined results came to a clear conclusion that the compounds could interfere with the whole intracellular cycle of $C$. trachomatis, mainly targeting the middle $\mathrm{RB}$ proliferation periods. Our findings suggest the potential of pyrroloisoxazolidine derivatives as lead molecules for the development of antichlamydial agents. Additional animal studies were required to verify the compounds' antichlamydial activity in vivo. 


\section{Data Availability}

The data used to support the findings of this study are included within the article.

\section{Conflicts of Interest}

The authors declare no conflict of interest.

\section{Authors' Contributions}

Min Ni and Shunxin Xu contributed equally to this work.

\section{Acknowledgments}

This work was supported by the Science and Technology Project Fund of Nantong City (JC2019097 to XB), the Fifth 226 High Level Talent Training Project of Nantong City to $\mathrm{XB}$, and the National Natural Science Foundation of China (Grant No. 31400165 and 31370209 to XB).

\section{References}

[1] C. Elwell, K. Mirrashidi, and J. Engel, "Chlamydia cell biology and pathogenesis," Nature Reviews Microbiology, vol. 14, no. 6, pp. 385-400, 2016.

[2] S. Banhart, L. Rose, L. Aeberhard, S. Koch-Edelmann, and D. Heuer, "Chlamydia trachomatis and its interaction with the cellular retromer," International Journal of Medical Microbiology, vol. 308, no. 1, pp. 197-205, 2018.

[3] F. Bayramova, N. Jacquier, and G. Greub, "Insight in the biology of_Chlamydia_-related bacteria," Microbes and Infection, vol. 20, no. 7-8, pp. 432-440, 2018.

[4] H. R. Taylor, M. J. Burton, D. Haddad, S. West, and H. Wright, “Trachoma," Lancet, vol. 384, no. 9960, pp. 2142-2152, 2014.

[5] H. Fan, "Blindness-causing trachomatous trichiasis biomarkers sighted," Investigative ophthalmology \& visual science, vol. 53, no. 6, article 2560, 2012.

[6] K. Hybiske and R. S. Stephens, "Mechanisms of Chlamydia trachomatis entry into nonphagocytic cells," Infection and Immunity, vol. 75, no. 8, pp. 3925-3934, 2007.

[7] K. Hybiske and R. S. Stephens, "Mechanisms of host cell exit by the intracellular bacterium Chlamydia," Proceedings of the National Academy of Sciences of the United States of America, vol. 104, no. 27, pp. 11430-11435, 2007.

[8] K. M. Sandoz and D. D. Rockey, "Antibiotic resistance in Chlamydiae," Future Microbiology, vol. 5, no. 9, pp. 14271442, 2010.

[9] K. A. Workowski, "Centers for disease control and prevention sexually transmitted diseases treatment guidelines," Clinical Infectious Diseases, vol. 61, Supplement 8, pp. S759-S762, 2015.

[10] N. Borel, C. Leonard, J. Slade, and R. V. Schoborg, "Chlamydial antibiotic resistance and treatment failure in veterinary and human medicine," Current Clinical Microbiology Reports, vol. 3, no. 1, pp. 10-18, 2016.

[11] A. R. Bhengraj, H. Vardhan, P. Srivastava, S. Salhan, and A. Mittal, "Decreased susceptibility to azithromycin and doxycycline in clinical isolates of Chlamydia trachomatis obtained from recurrently infected female patients in India," Chemotherapy, vol. 56, no. 5, pp. 371-377, 2010.
[12] J. Gieffers, J. Rupp, A. Gebert, W. Solbach, and M. Klinger, "First-choice antibiotics at subinhibitory concentrations induce persistence of Chlamydia pneumoniae," Antimicrobial Agents and Chemotherapy, vol. 48, no. 4, pp. 1402-1405, 2004.

[13] D. C. W. Mabey, V. Hu, R. L. Bailey, M. J. Burton, and M. J. Holland, "Towards a safe and effective chlamydial vaccine: lessons from the eye," Vaccine, vol. 32, no. 14, pp. 15721578, 2014.

[14] G. Zhong, R. C. Brunham, L. M. de la Maza, T. Darville, and C. Deal, "National Institute of Allergy and Infectious Diseases workshop report: "Chlamydia vaccines: The way forward"," Vaccine, vol. 37, no. 50, pp. 7346-7354, 2019.

[15] M. N. Starnbach, "Action Needed on _Chlamydia_ Vaccines," Trends in Microbiology, vol. 26, no. 8, pp. 639-640, 2018.

[16] S. Marwaha, H. Uvell, O. Salin et al., "N-Acylated derivatives of sulfamethoxazole and sulfafurazole inhibit intracellular growth of Chlamydia trachomatis," Antimicrobial Agents and Chemotherapy, vol. 58, no. 5, pp. 2968-2971, 2014.

[17] X. Bao, A. Gylfe, G. L. Sturdevant et al., "Benzylidene acylhydrazides inhibit chlamydial growth in a type III secretionand iron chelation-independent manner," Journal of Bacteriology, vol. 196, no. 16, pp. 2989-3001, 2014.

[18] U. R. Abdelmohsen, C. Cheng, A. Reimer et al., "Antichlamydial sterol from the Red Sea sponge Callyspongia aff. implexa," Planta Medica, vol. 81, no. 5, pp. 382-387, 2015.

[19] J. A. D. Good, J. Silver, C. Núñez-Otero et al., “Thiazolino 2-pyridone amide inhibitors ofChlamydia trachomatisInfectivity," Journal of Medicinal Chemistry, vol. 59, no. 5, pp. 2094-2108, 2016.

[20] J. L. Li, D. Chen, L. Huang et al., "Antichlamydial dimeric indole derivatives from marine actinomycete Rubrobacter radiotolerans," Planta Medica, vol. 83, no. 9, pp. 805-811, 2017.

[21] I. M. Petyaev, N. A. Zigangirova, E. Y. Morgunova, N. H. Kyle, E. D. Fedina, and Y. K. Bashmakov, "Resveratrol inhibits propagation of Chlamydia trachomatis in McCoy cells," BioMed Research International, vol. 2017, Article ID 4064071, 7 pages, 2017.

[22] J. L. Li, N. Yang, L. Huang et al., "Pyocyanin inhibits Chlamydia infection by disabling infectivity of the elementary body and disrupting intracellular growth," Antimicrobial Agents and Chemotherapy, vol. 62, no. 6, p. e02260, 2018.

[23] K. M. Gallegos, C. R. Taylor, D. J. Rabulinski et al., “A synthetic, small, sulfated agent is a promising inhibitor of Chlamydia spp. infection in vivo," Frontiers in Microbiology, vol. 9, 2019.

[24] M. A. Brown, M. G. Potroz, S. W. Teh, and N. J. Cho, "Natural products for the treatment of chlamydiaceae infections," Microorganisms, vol. 4, no. 4, p. 39, 2016.

[25] E. Karhu, J. Isojärvi, P. Vuorela, L. Hanski, and A. Fallarero, "Identification of privileged antichlamydial natural products by a ligand-based strategy," Journal of Natural Products, vol. 80, no. 10, pp. 2602-2608, 2017.

[26] S. Cecioni, K. Aouadi, J. Guiard et al., "Novel routes to either racemic or enantiopure $\alpha$-amino-(4-hydroxy- pyrrolidin-3yl)acetic acid derivatives and biological evaluation of a new promising pharmacological scaffold," European Journal of Medicinal Chemistry, vol. 98, pp. 237-249, 2015.

[27] S. Ghannay, S. Bakari, M. Msaddek, A. Kadri, K. Aouadi, and S. Vidal, "Design, synthesis, molecular properties and in vitro antioxidant and antibacterial potential of novel enantiopure 
isoxazolidine derivatives," Arabian Journal of Chemistry, vol. 13, no. 1, pp. 2121-2131, 2020.

[28] S. Ghannay, S. Bakari, A. Ghabi, A. Kadri, M. Msaddek, and K. Aouadi, "Stereoselective synthesis of enantiopure Nsubstituted pyrrolidin-2, 5-dione derivatives by 1,3-dipolar cycloaddition and assessment of their in vitro antioxidant and antibacterial activities," Bioorganic \& Medicinal Chemistry Letters, vol. 27, no. 11, pp. 2302-2307, 2017.

[29] B. Loh, L. Vozzolo, B. J. Mok et al., "Inhibition of HIV-1 replication by isoxazolidine and isoxazole sulfonamides," Chemical Biology \& Drug Design, vol. 75, no. 5, pp. 461474, 2010.

[30] K. R. R. Kumar, H. Mallesha, and K. S. Rangappa, "Synthesis of novel isoxazolidine derivatives and their antifungal and antibacterial properties," Archiv der Pharmazie, vol. 336, no. 3, pp. 159-164, 2003.

[31] Y. F. Kong, Y. Z. Liu, B. Y. Wang et al., "The catalystcontrolled divergent cascade reactions of homo-propargylic amines and nitrones: synthesis of pyrrolo-isoxazolidines and $\gamma$-lactams," Advanced Synthesis \& Catalysis, vol. 360, no. 6, pp. 1240-1252, 2018.

[32] S. L. McGovern, B. T. Helfand, B. Feng, and B. K. Shoichet, "A specific mechanism of nonspecific inhibition," Journal of Medicinal Chemistry, vol. 46, no. 20, pp. 4265-4272, 2003.

[33] S. L. Mcgovern, E. Caselli, N. Grigorieff, and B. K. Shoichet, "A common mechanism underlying promiscuous inhibitors from virtual and high-throughput screening," Journal of Medicinal Chemistry, vol. 45, no. 8, pp. 1712-1722, 2002.

[34] H. Ji, C. Wu, M. Ni et al., "In vitro antichlamydial activity of 1 , 2, 3, 5-tetrasubstituted pyrrole derivatives," Chemotherapy, vol. 63, no. 2, pp. 95-99, 2018.

[35] M. M. Cosse, R. D. Hayward, and A. Subtil, "One face of Chlamydia trachomatis: the infectious elementary body," Current Topics in Microbiology and Immunology, vol. 412, no. 4356, pp. 35-58, 2018. 